\section{Vom Nachzügler zum Vorreiter?}

\section{Eine der Hauptaufgaben einer neugewählten Bundesregierung wird die Ein- führung einer ökologischen Steuerreform sein. Gerne wird in diesem Zusam- menhang auf die Vorreiter in den Nachbarländern verwiesen. Eine genavere Analyse führt zu aufschlußreichen Ergebnissen.}

I

Von Jan Nill

nzwischen haben mindestens acht Länder _Kohlendioxid-/ Energiesteuern eingeführt (1). Bei drei bis fünf von ihnen kann von einer wirklichen Ökologischen Steuerreform gesprochen werden, d.h. einer gezielten Belastung des Faktors Umweltverbrauch bei einer Entlastung an anderer Stelle. Den Anfang machte Schweden mit einer umfassenden Steuerreform bereits 1991, bei der u.a. die bestehende Energiesteuer erhöht und um eine Kohlendioxidkomponente erweitert wurde. Daneben wurden Stickstoff und Schwefeldioxid besteuert. Das Aufkommen betrug 1995 bereits 12 Mrd. Mark. 1996/97 fand eine Erweiterung und Verschärfung statt. In Dänemark begann, nachdem 1992 eine Kohlendioxid-Abgabe eingeführt wurde, die umfassende Reform 1994: Die Steuer wurde dynamisiert und auf Strom ausgeweitet, außerdem wurden Abfall, Wasser und Schwefeldioxid einbezogen; Ziel ist ein Anstieg des Anteils der Umweltsteuern am gesamten Steueraufkommen auf 15 Prozent. Seit 1996 wird die Industrie sukzessive einbezogen. In den Niederlanden wurde, eingebettet in das Konzept der nationalen Umweltplanung, 1992 eine Kohlendioxid-/ Energiesteuer eingeführt, 1996 wurde die Energiesteuer für Kleinverbraucher ausgeweitet. Weitere nennenswerte Ansätze bestehen in Norwegen und Finnland, die beide auch bereits Anfang der 90er Jahre in die Kohlendioxid-Besteuerung einstiegen und sie 96/97 ausweiteten. Was die Höhe und den Umfang der Besteuerung betrifft, ragen jedoch Dänemark und Schweden heraus (2).

Einer der umstrittensten Punkte ist der Einbezug der Industrie in die Besteuerung. Die ökologische und ökonomische Effizienz sprechen eindeutig dafür, doch werden Gefahren für die Wettbewerbsfähigkeit befürchtet. Die bisherigen Vorreiter sind kleine, offene Länder, und damit diesem Argument sehr zugänglich. Tatsächlich erheben alle auf energieintensive Branchen nur sehr geringe Abgaben. Während Norwegen und Finnland einheitliche, aber nominal niedrige Steuersätze einführten, läßt sich in Dänemark und Schweden nur ein sehr zögerlicher Einbezug der Industrie beobachten; Schweden nahm - mit schnell deutlich werdenden negativen ökologischen Konsequenzen - die anfängliche Belastung der Industrie bereits 1993 weitgehend zurück und schichtete deren Anteil auf die Haushalte um. 1996 wurde mit Ausnahme der Stromproduzenten und stark belasteter Industrien die Steuer für die Industrie wieder auf 50 Prozent des allgemeinen Steuersatzes erhöht. In Dänemark war die Industrie bis 1996 faktisch vollständig von der Besteuerung ausgenommen; bis 2000 erfolgt jetzt jedoch eine schrittweise, jedoch stark nach dem Grad der Energieintensität der Branche differenzierte, Integration, wobei über Zuschüsse für Energiesparmaßnahmen und Senkung der Sozialversicherungsbeiträge eine Rückerstattung erfolgen soll. Zudem ist auffällig, daß alle nordischen Länder trotz Ökosteuer durch erheblich niedrigere industrielle Energiepreise (insbesondere für Strom und Heizöl) als in Mittel- und vor allem Südeuropa gekennzeichnet sind (3). Auch bei der gerade in Deutschland heiß umstrittenen Mineralölsteuer liegen diese Länder im OECDVergleich eher am unteren Ende; der Verkehrsbereich ist bisher noch weitgehend von der Ökosteuer ausgeklammert.

Das Argument der Wettbewerbsfähigkeit genießt also selbst in den Vorreiterländern Priorität. Auch hat sich kein Land auf allein preisinduzierte "first-mover-advantages" verlassen, stattdessen. werden der Anpassungsprozeß sowie die umweltfreundlicheren Alternativen im Energiebereich in der Regel subventioniert. Angesichts der bisher relativ geringen Höhe der Besteuerung im Produktionsbereich kann dies allerdings kaum verwundern. Somit ist bezüglich der Einleitung eines wirklichen ökologischen Strukturwandels durchaus noch Spielraum für weitere Vorreiter gegeben.

Was den politischen Prozeß der Einführung betrifft, so hat sich bisher, gerade auch angesichts der Widerstände bei den potentiellen Ver- liererbranchen, ein graduelles und eher vorsichtiges Vorgehen unter Einbezug der Industrie als erfolgversprechend erwiesen. Das Paradebeispiel ist wiederum Dänemark, wo in Stufen vorgegangen und zudem jeweils über einen Zeitraum von fünf Jahren ein Dynamisierungspfad festgelegt wurde. Aus der Einführung entstand insofern eine endogene Dynamik, als schnell deutlich wurde, daß die angestrebten umweltpolitischen Ziele ohne eine Steuererhöhung nicht zu erreichen waren. Als Negativbeispiele können Schweden und Finnland genannt werden. Schweden sah sich genötigt, in der Rezession von 1992/93 einen Teil der ehrgeizigen Pläne auf Druck der Industrie zurückzunehmen. Finnland geriet trotz niedriger nominaler Steuersätze ob seines Einbezugs der Industrie unter solchen politischen Druck, daß es in der Folge ebenfalls einen Teil der Belastung auf die Haushalte umschichtete.

Als politische Erfolgsbedingung weniger relevant ist hingegen die Industriestruktur. Zwar gibt es Differenzen der Marktstruktur des Energiesektors, aber sowohl die Niederlande als auch Schweden haben tendenziell eine ähnliche, und sogar energieintensivere, Industriestruktur wie Deutschland.

Schließlich zeigt sich, daß es paradoxerweise eher interventionistisch gesonnene Regierungen waren, die das marktnahe Instrument der Ökosteuern vorantrieben. Eine Regierungsbeteiligung der Sozialdemokraten erwies sich dabei in aller Regel als förderlich.

\section{Anmerkungen}

(1) OECD: Environmental Taxes and Green Tax Reform, Paris 1997, S. 52 (Stand März 1997).

(2) Vgl. für detailliertere Angaben u.a.

Martin Jänicke, Lutz Mez et al.: Ökologische und wirtschaftliche Aspekte einer Energiebesteuerung im internationalen Vergleich. FFU-rep 98-2, S. 7ff;

P. Malaska et al.: Environment-Based Energy Taxation in the Nordic Countries. Ministry of the Environment, Helsinki 1997; International Energy Agency: Energy Policies of IEA Countries. 1997 Review

(3) OECD: Implementation Strategies for Environmental Taxes, Paris 1996, Annex 1 und 2.

\section{Der Autor}

Jan Nill ist verantwortlicher Redakteur von Ökologisches Wirlschaften.

Kontakt: Institut für ökologische Wirischaftsforschung (IÖW), Giesebrechtstr. 13, 10629 Berlin, Tel. 030/884594-28, Fax 030/8825439, E-mail: jan.nill@ioew.b.eunet.de 
(c) 20I0 Authors; licensee IÖW and oekom verlag. This is an article distributed under the terms of the Creative Commons Attribution Non-Commercial No Derivates License (http://creativecommons.org/licenses/by-nc-nd/3.o/), which permits unrestricted use, distribution, and reproduction in any medium, provided the original work is properly cited. 